\title{
Grazing impact oscillations
}

\author{
John de Weger, ${ }^{1}$ Willem van de Water, ${ }^{1}$ and Jaap Molenaar ${ }^{2}$ \\ ${ }^{1}$ Physics Department, Eindhoven University of Technology, P.O. Box 513, 5600 MB Eindhoven, The Netherlands \\ ${ }^{2}$ Mathematics Department, Eindhoven University of Technology, P.O. Box 513, 5600 MB Eindhoven, The Netherlands
}

(Received 27 December 1999)

\begin{abstract}
An impact oscillator is a periodically driven system that hits a wall when its amplitude exceeds a critical value. We study impact oscillations where collisions with the wall are with near-zero velocity (grazing impacts). A characteristic feature of grazing impact dynamics is a geometrically converging series of transitions from a nonimpacting period- 1 orbit to period- $M$ orbits that impact once per period with $M=1,2, \ldots$ In an experiment we explore the dynamics in the vicinity of these period-adding transitions. The experiment is a mechanical impact oscillator with a precisely controlled driving strength. Although the excitation of many high-order harmonics in the experiment appeared unavoidable, we characterize it with only three parameters. Despite the simplicity of this description, good agreement with numerical simulations of an impacting harmonic oscillator was found. Grazing impact dynamics can be described by mappings that have a square-root singularity. We evaluate several mappings, both for instantaneous impacts and for impacts that involve soft collisions with a yielding wall. As the square-root singularity appears persistent in the reduction of the dynamics to mappings, and because impact dynamics appears insensitive to experimental nonidealities, the characteristic bifurcation scenario should be observed in a wide class of experimental systems.
\end{abstract}

PACS number(s): 05.45.-a, 46.40.-f, 46.55.+d

\section{INTRODUCTION}

An impact oscillator is a periodically driven oscillator that impacts with a wall when its excursion exceeds a critical value. In between impacts its dynamics can be linear, but it inherits a strong nonlinearity from the mere presence of the wall. In its simplest guise, the impact can be an instantaneous velocity reflection with a coefficient of restitution representing the energy loss during impact. A grazing impact is an impact with zero velocity. Grazing impacts sensitively depend on initial conditions: small variations in initial conditions can cause the oscillator to cross the border between colliding and noncolliding orbits.

The model system that we consider is sketched in Fig. 1. A harmonic oscillator with mass $m$ and spring constant $K$ is driven sinusoidally with amplitude $F$ and frequency $\omega$. Its linear damping is gauged by $\nu$. When its deflection becomes larger than $d$, it collides with the wall. This wall may actually be elastic. Between impacts, the equation of motion for the oscillator is

$$
m \ddot{x}+\nu \dot{x}+K(x+d)=F \cos (\omega t+\phi) .
$$

Impact oscillators display a perplexing variety of nonlinear behavior such as chaos, subharmonic resonances, and period doublings [1], but a strong organizing principle has been lacking for a long time. Grazing impacts are special because near grazing orbits the dynamical system can be reduced to iterations of a mapping. Nordmark [2] showed how to reach such a reduced description for arbitrary periodically driven systems that are close to grazing. The derivation of mappings for special values of the system parameters was described in [3]. In impact maps, a borderline separates nonimpacting from impacting orbits. For a nonimpacting orbit the map is linear, but it has a square-root singularity for an impacting orbit. Close to grazing, the accel- eration near impact can be considered constant and the square root is simply the relation between elapsed time and traveled distance in systems with constant acceleration. The square-root singularity and the associated extreme stretching of phase space near the point of grazing impact lead to highly nontrivial dynamics.

As the bifurcation properties of a mapping are analyzed much more readily than those of a differential equation, the study of these mappings led to the discovery of important organizing principles in the dynamics of impact oscillators. A near-exhaustive examination of the bifurcations of the Nordmark map [2] was reported by Chin et al. [4]. This work has inspired our research. The bifurcation structure found is exemplary for a more general class of bordercollision bifurcations that arise in nonsmooth systems [6].

One of the predictions in [4] is the occurrence of a series of transitions from a nonimpacting period-1 $\left(p_{1}\right)$ orbit to period- $M\left(p_{M}\right)$ orbits with $M=3,4, \ldots$. We will call this a series of period-adding transitions. The $M$-periodic orbits have one impact per period and are termed maximal periodic orbits. As will also be explained in Sec. IC, these transitions

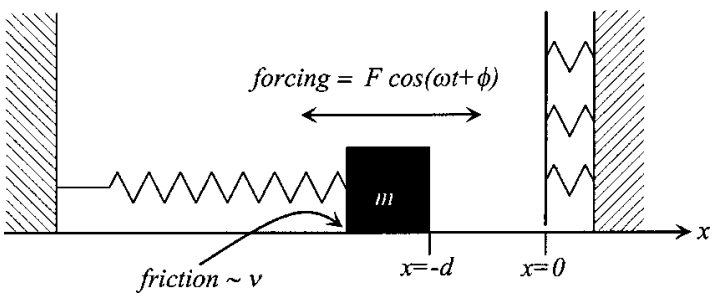

FIG. 1. The physical system studied in this paper. The mass $m$ of the oscillator can collide with a yielding wall. We assume that the massless wall is attached with frictionless springs to the fixed world. The resilience of the wall is determined by the stiffness of these springs. The wall position in rest is at $x=0$ and the rest position of the oscillating mass at $x=-d$. 
are intimately tied to the square-root singularity of impact dynamics [5]. It is tempting to draw the analogy with the universal series of period doublings that exist in maps with a quadratic nonlinearity.

No practical physical system can display a singularity, and the question is whether period adding transitions can be observed in a real experiment. In practical systems, collisions will be with a more or less yielding wall, which induces a time delay at impact. Noninstantaneous impacts may also result from the excitation of high-order modes of the oscillator, which is hard to avoid in an experiment. The question is if such time delays will effectively smooth the singularity, thus essentially altering the bifurcation structure of the impact oscillator. To answer these important questions, we have in [7] rederived grazing impact maps for collisions, with both perfectly rigid and yielding walls. It turns out that in almost all cases the square-root singularity persists in the mapping.

The challenge of the experiment is to see if a singularity survives the intrinsic nonidealities of the experimental setup. In this paper we will describe the results of experiments aimed at measuring the geometrically progressing periodadding transitions $p_{1} \leftrightarrow p_{M}, M=2,3, \ldots$. We will focus on underdamped oscillators as these are most relevant to mechanical experiments. A transition from the nonimpacting period-1 orbit to a $p_{1}$ orbit with one grazing impact was experimentally observed by Thompson, Bishop, and Foale [9]; our highest observed period is $M=10$. In order to see such high-order resonances, precise control of the excitation is needed. Our setup is described in Sec. II. We will seek a description of our experimental oscillator in terms of only three parameters: the period $T$ of its free oscillations, its linear damping $\lambda$, and the coefficient of restitution $r$, which represents the collision energy loss. In Sec. III we show that numerical simulations of an impacting harmonic oscillator with these parameters as input agree well with the experiment. Favorable agreement between experiments and numerical simulations (but in a different region of parameter space) was also found by Shaw [10], who studied impact oscillations with hard impacts and who coped with the same experimental nonidealities, such as the excitation of many high-order modes.

In order to explore the dynamics away from grazing impacts, we have scanned phase space in the vicinity of maximal periodic orbits. Here also we find favorable agreement with the result of numerical simulations. In these experiments, a large variety of periodic and chaotic states was encountered. This emphasizes that impact dynamics is extremely rich, but it also stresses the necessity of bringing order to this area. As we argued, such order is now provided by the reduction of the dynamical system to an iterated map. A first account of our experimental results appeared in [8].

The differential equation Eq. (1) of an impact oscillator can be reduced to a mapping for orbits that remain close to grazing ones. As the reduction is highly nontrivial, it is important to test the prediction of the mappings quantitatively against those of the original differential equation, especially if one moves away from strictly grazing orbits. Such a comparison will be made in Sec. IV for the sequence of period addings. It turns out that mappings, both for instantaneous collisions with a rigid wall and for soft collisions with a yielding wall, capture the essence of impact dynamics.

\section{A. The harmonic oscillator}

If we scale time with the period of the external driving and the deflection $x$ with the distance $d$ to the wall, Eq. (1) becomes in dimensionless units

$$
\ddot{x}+\widetilde{\nu} \dot{x}+\Omega^{2}(x+1)=\widetilde{F} \cos (2 \pi t+\phi),
$$

where $\quad \tilde{\nu}=2 \pi \nu / m \omega, \quad \Omega^{2}=4 \pi^{2} K / m \omega^{2}, \quad$ and $\quad \tilde{F}$ $=4 \pi^{2} F / m \omega^{2} d$; from now on we will drop the tilde on the transformed quantities. In the case of collisions with a flexible wall the harmonic force $\Omega^{2} x$ is replaced by $K(x)$,

$$
\ddot{x}+\nu \dot{x}+K(x)=F \cos (2 \pi t+\phi),
$$

with

$$
K(x)= \begin{cases}\Omega^{2}(x+1), & x \leqslant 0 \\ \Omega^{2}(x+1+\Gamma x), & x>0,\end{cases}
$$

where $\Gamma$ is the ratio of the spring constant of the resilient wall to that of the oscillator. The noncolliding oscillator just grazes the wall when the driving amplitude and phase are

$$
\begin{gathered}
F_{g}=\left[\left(\Omega^{2}-4 \pi^{2}\right)^{2}+4 \pi^{2} \nu^{2}\right]^{1 / 2} \text { and } \\
\phi=\cos ^{-1}\left[\left(\Omega^{2}-4 \pi^{2}\right) / F_{g}\right],
\end{gathered}
$$

which we use to define a normalized driving strength $\sigma$ $=\left(F-F_{g}\right) / F_{g}$. It is also convenient to introduce a normalized driving frequency $\xi$ as $\xi=f T$, where $T=2 \pi /\left[\Omega^{2}\right.$ $\left.-(\nu / 2)^{2}\right]^{1 / 2}$ is the period of the free damped oscillations of the oscillator and $f=\omega / 2 \pi$ is the excitation frequency.

In the case of an infinitely stiff wall $(\Gamma=\infty)$, the mass will not penetrate the wall but reflect instantaneously. When the position $x(t)$ comes to the boundary at, say, $t_{0}$ the velocity reflects as $\dot{x}\left(t_{0^{+}}\right)=-r \dot{x}\left(t_{0^{-}}\right)$, where $r$ is the restitution coefficient and $\dot{x}\left(t_{0^{-}}\right)=v_{c}$ is the collision velocity. For this case Nordmark [2] was able to reduce the dynamics of the impact oscillator to that of a simple two-dimensional mapping.

$$
\begin{aligned}
& x_{n+1}=\alpha x_{n}+y_{n}+\rho, \quad \text { if } x_{n} \leqslant 0, \\
& y_{n+1}=-\gamma x_{n} \\
& x_{n+1}=-\sqrt{x_{n}}+y_{n}+\rho, \quad \text { if } x_{n}>0, \\
& y_{n+1}=-\gamma r^{2} x_{n}
\end{aligned}
$$

where $x_{n}$ and $y_{n}$ are transformed coordinates of the $(x, \dot{x})$ space at stroboscopic times $t_{n}=n$, and where $\rho$ is the bifurcation parameter that measures the distance to the point of grazing impact. If no collision occurs between $t_{n}$ and $t_{n+1}$, the linear map Eq. (5) applies, whereas Eq. (6) describes the dynamics in the case that an impact will occur on $\left[t_{n}, t_{n+1}\right]$. For oscillators described by Eq. (2), the parameters of the mapping can be expressed explicitly in those of the differential equation. This is done most conveniently in terms of the time-1 operator $\mathbf{P}$ which connects states of the noncolliding oscillator at successive stroboscopic times,

$$
\mathbf{P}=\frac{1}{s_{2}-s_{1}}\left(\begin{array}{cc}
s_{2} e^{s_{1}}-s_{1} e^{s_{2}} & e^{s_{2}}-e^{s_{1}} \\
s_{1} s_{2}\left(e^{s_{1}}-e^{s_{2}}\right) & s_{2} e^{s_{2}}-s_{1} e^{s_{1}}
\end{array}\right),
$$


where $\quad s_{1}=\frac{1}{2}\left(-\nu+\sqrt{\nu^{2}-4 \Omega^{2}}\right) \quad$ and $\quad s_{2}=\frac{1}{2}(-\nu$ $\left.-\sqrt{\nu^{2}-4 \Omega^{2}}\right)$. The parameters $\alpha$ and $\gamma$ of the collisionless linear map Eq. (5) are in a simple way related to the eigenvalues $e^{s_{1}}$ and $e^{s_{2}}$ of $\mathbf{P}$ as [4]

$$
\begin{gathered}
\alpha=e^{s_{1}+e^{s_{2}}}=2 e^{-\nu / 2} \cosh \left(\frac{1}{2} \sqrt{\nu^{2}-4 \Omega^{2}}\right), \\
\gamma=e^{s_{1}} \times e^{s_{2}}=e^{-\nu},
\end{gathered}
$$

which expresses the fact that the eigenvalues of $\mathbf{P}$ must be the same as those of the Jacobian of Eq. (5). The parameters of the nonlinear collision map Eq. (6) and the scaling of the bifurcation parameter $\rho$ can only be obtained from a full nonlinear analysis, in particular,

$$
\rho=\frac{1-\alpha+\gamma}{8 \pi^{2}(1+r)^{2} \mathbf{P}_{12}^{2}} \sigma,
$$

which for underdamped oscillators $\left(\nu^{2}<4 \Omega^{2}\right)$ can be written in terms of the reduced driving frequency $\xi$ as

$$
\rho=\frac{1-\alpha+\gamma}{2 \gamma \xi^{2}(1+r)^{2} \sin ^{2}(2 \pi / \xi)} \sigma .
$$

The mapping contained in Eqs. (5) and (6) also applies to periodically driven systems other than the harmonic oscillator but in these cases the quantitative association of the map parameters with the physical parameters is much more difficult. The presence of the square root in Eq. (6) is a key aspect of the mapping; it causes the Jacobian to be singular at $x_{n}=0$. We will explain below that it gives rise to the characteristic period-adding bifurcations that will be studied in this paper.

The reduction of grazing impact dynamics described by a differential equation to a simple mapping rests on the assumptions that the actual orbit stays close to the nonimpacting grazing orbit, that the driving amplitude stays close to the grazing amplitude $F_{g}(|\sigma| \ll 1)$, and that the collision velocity remains small $\left(\left|v_{c}\right| \ll 1\right)$. For strictly grazing orbits, the map is exact. Careful analysis is needed to derive mappings that are also valid in the vicinity of grazing orbits, and that can be used to explore the bifurcation structure of grazing impact oscillators.

In [7] we rederived mappings from the differential equation Eq. (3), but now also allowing for a finite stiffness of the wall. Our derivation has followed a slightly different route than in [2], with for the infinitely stiff wall the result

$$
\begin{aligned}
& x_{n+1}=\alpha x_{n}+y_{n}+\rho, \quad \text { if } x_{n} \leqslant 0, \\
& y_{n+1}=-\gamma x_{n} \\
& x_{n+1}=-c_{1} \sqrt{x_{n}}+c_{2} x_{n}+y_{n}+\rho, \quad \text { if } x_{n} \geqslant 0, \\
& y_{n+1}=c_{3} x_{n}
\end{aligned}
$$

The coefficients $c_{1}, c_{2}$, and $c_{3}$ are now given by

$$
\begin{gathered}
c_{1}=\operatorname{sgn}\left(\mathbf{P}_{12}\right), \\
c_{2}=\alpha-2(1+r) \mathbf{P}_{22}+(1+r)^{2} \mathbf{P}_{22}^{2}, \\
c_{3}=(1+2 r) \gamma-(1+r)^{2} \mathbf{P}_{22}^{3} .
\end{gathered}
$$

There are slight but significant differences between the maps Eqs. (5),(6) and Eqs. (10),(11); those were discussed extensively in [7]. For example, the sign factor in Eq. (12), which is absent in the Nordmark map Eq. (6), is needed to explain period-1 impacting orbits. In Sec. IV we will argue that the term proportional to $x_{n}$ in Eq. (11), which is also absent in the Nordmark map, is needed to correctly predict the loss of stability of maximal periodic orbits due to an additional impact.

The map Eqs. (10)-(12) applies to impacts with an infinitely stiff wall $(\Gamma=\infty)$. In [7] we also derived a map for yielding walls with a finite value of $\Gamma$. In the case of a resilient wall, collisions may be soft or hard. The parameter that distinguishes these two cases is $\beta=r v_{c} \Gamma^{1 / 2} \Omega / 4 \pi^{2}$ where $v_{c}=\dot{x}\left(t_{0^{-}}\right)$is the collision velocity. Collisions are hard if $\beta \gg 1$; then the map Eqs. (10)-(12) applies. Collisions are soft if $\beta \ll 1$; then the same map Eqs. (10)-(12) applies, but now with the substitution $r \rightarrow-r$. Therefore, both hard and soft collisions involve the square-root singularity. Although we were unable to find a mapping that pertains to the intermediate case, we believe that the square-root behavior applies to all collisions.

In the soft maps, the restitution rule is used as $\dot{x}\left(t_{0^{+}}\right)$ $=r \dot{x}\left(t_{0^{-}}\right) \quad\left(=r v_{c}\right)$. An exception to the square-root rule arises for soft collisions that are perfectly elastic $(r=1)$. It appears that in this case the presence of the wall can be completely ignored to within the order of the approximations used. Accordingly, these impact oscillations have a completely different bifurcation structure.

\section{B. Numerical integration}

A numerical simulation of the differential equation Eqs. (3),(4) in the presence of grazing impacts needs to be done with care. It is absolutely crucial not to miss boundary crossings of $x(t)$. A practical and efficient way is to compute the positions $x(t)$ and velocities $\dot{x}(t)$ at a small number of discrete points $t_{1}, \ldots, t_{k}$ in each drive period, either by using the exact solution [Eq. (7)] or by numerically integrating the differential equation. Boundary crossings are found by checking if the series $x\left(t_{1}\right), \ldots, x\left(t_{k}\right)$ crosses $x=0$. If so, the precise crossing instant is determined to machine precision using a zero finding procedure. At this point, the switch of Eq. (4) can be made.

Checking the computed positions $x\left(t_{1}\right), \ldots, x\left(t_{k}\right)$ is not sufficient, as a boundary crossing may go unnoticed. Therefore, we also keep track of the turning points where the velocity $\dot{x}=0$. The turning instants $t_{t}$ can be found by looking for zero crossings of $\dot{x}\left(t_{1}\right), \ldots, \dot{x}\left(t_{k}\right)$ and pinpointing the precise turning point using a zero finding procedure. At each turning point it is checked if $x\left(t_{t}\right)>0$, which signifies a boundary crossing.

The number $k$ of discrete points in the regions $x<0$ and $x>0$ is taken proportional to $\Omega$ and $\Gamma^{1 / 2} \Omega$, respectively. If the differential equations are numerically integrated, zeros can be found efficiently and accurately using a trick described by Hénon [12].

\section{Period addings}

A period- $M$ maximal periodic orbit impacts only once in every $M$ periods of the driving. When the stroboscopic phase 

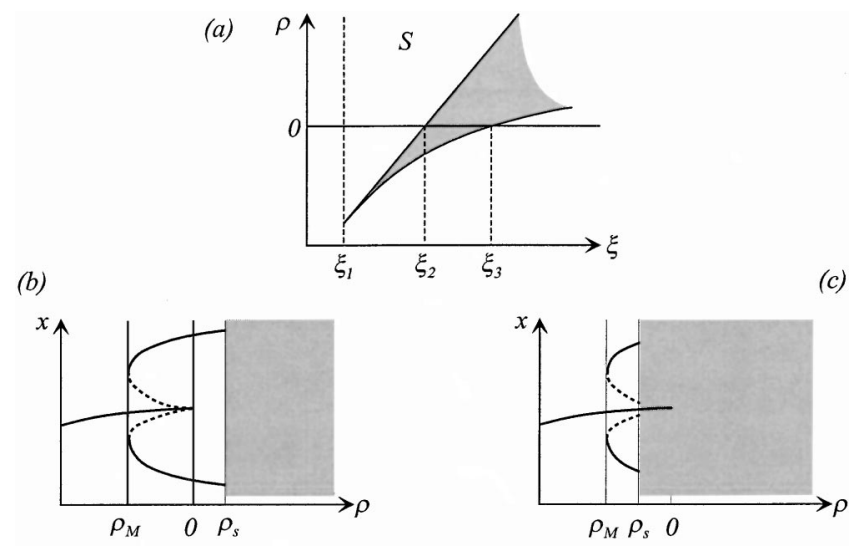

FIG. 2. Period-adding bifurcations. Shown are $p_{1} \leftrightarrow p_{M}$ transitions $(M=2)$ at various values of the normalized excitation frequency $\xi$. The $p_{1}$ orbit is the nonimpacting oscillator. (a) Stability tongue in the $\rho, \xi$ plane. At its upper boundary the $p_{M}$ orbit loses stability to states $S$ through a second impact. At the lower boundary the $p_{M} \rightarrow p_{1}$ transition is observed in a quasistatic downward scan of $\rho$. (b) Bifurcation diagram of a $p_{2}$ orbit obtained in a quasistatic upward and downward scan of $\rho$ at excitation frequencies $\xi_{2}<\xi$ $<\xi_{3}$. The dashed line indicates the unstable $p_{2}$ branch. The shaded area indicates the transition to states $(S)$ with a second impact. (c) Same as (b), but now $\xi_{1}<\xi<\xi_{2}$.

is chosen such that $x>0$ is impacting and $x<0$ is not (as it is in the mapping), the sequence of oscillator positions satisfies $x_{1}<x_{2}<\cdots<x_{M-1}<0<x_{M}$. Period- $M$ maximal periodic orbits form a nice testing ground for a reduced description in terms of a mapping. These orbits exhibit a geometric progression toward $M=\infty$ in which the intervals of stability become geometrically narrower and in which the impact velocity vanishes as $M \rightarrow \infty$. The lower the period $M$, therefore, the more the assumptions of the map reduction are challenged.

A period adding in the underdamped oscillator is a saddle-node bifurcation, that is, the simultaneous creation of a stable and an unstable period- $M$ maximal periodic orbit at a negative value of $\rho=-\rho_{M} \leqslant 0[4,11]$. This situation is sketched in Fig. 2. At large negative $\rho$ the wall is not felt, and the oscillations are free. In a slow quasistatic upward scan of $\rho$, collisions with the wall occur first at $\rho=0$. The system then traces the stable period- $M$ branches. When $\rho$ is subsequently decreased, these branches are followed to negative $\rho$ until the point $\rho=-\rho_{M}$, where the stable and unstable branches meet. At this point, which lies on the lower stability boundary, the oscillator impacts with zero velocity.

In a maximal period- $M$ orbit the excursion of the oscillator increases steadily during $M-1$ periods of the excitation until it collides at the $M$ th cycle and is thrown back to negative $x$. At the upper boundary of the stability diagram of Fig. 2 the period- $M$ orbit loses stability through an additional impact at time $(M-1) T$; we will denote this orbit as $p_{M / 2}$. The general shape of the stability tongue of a maximal $M$-periodic orbit is sketched in Fig. 2(a).

In our experiment, the bifurcation parameter $\rho$ is quasistatically increased from the nonimpacting state $(\rho<0)$ to $\rho$ $=0$ where impacts first occur. When $\rho$ is increased further, the maximal periodic orbit will collide a second time, which marks the upper boundary of the region of existence at $\rho$ $=\rho_{s}$. After perusing some of the states at even larger values of $\rho$, the bifurcation parameter is again slowly decreased until the impacts cease at $\rho=-\rho_{M} \leqslant 0$. As Fig. 2 illustrates, the appearance of measured bifurcation diagrams depends on where one crosses the stability diagram of Fig. 2(a). For scaled frequencies $\xi_{1}<\xi<\xi_{2}$ [Fig. 2(c)], the state $S$ that is encountered first at $\rho=0$ is already beyond the upper boundary of the stability tongue. In this case the period- $M$ maximal orbit is encountered only in a downward scan of $\rho$. The state $S$ may actually be disconnected from the period- $M$ orbit so that in practice the maximal $M$-periodic orbit can be reached only by disturbing the oscillator. Zero velocity collisions are encountered at $\rho=-\rho_{M}$. When $\xi_{2}<\xi<\xi_{3}$ [Fig. 2(c)] the period- $M$ maximal periodic orbit is the first state encountered at $\rho=0$.

The way that these apparently hysteretic maximal period orbits arise in impact systems with a square-root singularity can be understood simply. To this aim we write the map in matrix form,

$$
\begin{aligned}
& \mathbf{x}_{n+1}=\mathbf{A} \mathbf{x}_{n}+\boldsymbol{\rho} \text { if } x_{n} \leqslant 0, \\
& \mathbf{x}_{n+1}=C\left(\begin{array}{c}
\sqrt{x_{n}} \\
0
\end{array}\right)+\mathbf{B} \mathbf{x}_{n}+\boldsymbol{\rho} \text { if } x_{n}>0 \text {, }
\end{aligned}
$$

with $\boldsymbol{\rho}$ the column vector with components $(\rho, 0)$. If we choose as cycle element $\mathbf{x}_{1}$ the one with $x_{1}>0$, a maximal period- $M$ cycle has one application of the nonlinear map Eq. (14) and $M-1$ applications of the linear map Eq. (13). The cycle condition $\mathbf{x}_{M+1}=\mathbf{x}_{1}$ then gives

$$
\mathbf{A}^{M-1}\left(C \sqrt{x_{1}} \mathbf{e}_{1}+\mathbf{B} \mathbf{x}_{1}+\boldsymbol{\rho}\right)+\left(\sum_{i=0}^{M-2}(\mathbf{A})^{i}\right) \boldsymbol{\rho}=\mathbf{x}_{1},
$$

with $\mathbf{e}_{1}$ the unit vector $(1,0)$. The $y$ component of the vector equation Eq. (15) is linear and can be used to solve for $y_{1}$; the solution can then be substituted in the first component of Eq. (15). The resulting equation is quadratic in $\sqrt{x_{1}}$. If it has two real positive solutions, one of them turns out to be stable, the other one unstable. This is the situation drawn in Figs. 2(b) and 2(c). At $\rho=0$ the unstable root collides with the origin, and for $\rho>0$ a single relevant root remains. The lower boundary of the $M$-resonance tongue is defined by the coalescence of the two roots $\left(\sqrt{x_{1}}\right)_{1,2}$ at $\rho=-\rho_{M}$.

\section{EXPERIMENT}

The aim of the experiment is to measure precise bifurcation diagrams near grazing impact, and to explore the geometric convergence of series of period-adding bifurcations. To reach this goal, a precise control of the frequency and amplitude of the excitation is needed.

The experiment is sketched in Fig. 3. It consists of a U-shaped, brass leaf spring that is excited horizontally by means of a large electromagnetic exciter on which it is mounted. The beam has length $13 \mathrm{~cm}$, width $2 \mathrm{~cm}$, and is made of $0.2 \mathrm{~mm}$ thick material; its clamped ends are $2 \mathrm{~cm}$ apart. The U shape suppresses undesired torsional motion of the beam. When the deflection of the beam is large enough, a ceramic ball that is attached to the beam collides with a hardened steel plate on the exciter. These materials are cho- 




FIG. 3. Experimental setup. A U-shaped leaf spring is brought into oscillation by horizontally oscillating its support. At a large enough forcing amplitude $F$, the attached mass impacts with a stop. Collisions take place between a hard ceramic ball and a hardened steel plate. The amplitude of the exciter is measured interferometrically. The deflection of the leaf spring is registered by reflecting a laser beam off the spring onto a position-sensitive diode.

sen such that the wear due to frequent impacts is negligible, so that the distance between the stop and the equilibrium position of the beam is constant. A problem in this experiment is the excitation of many higher harmonic modes upon impact. To increase the damping of these, adhesive tape is glued on the inner side of the spring and on the side that faces the exciter. The upper side of the spring is kept shiny for the measurement of the deflection of the spring using a laser beam.

The harmonic displacement of the beam support is realized by feeding a sine wave from a precision waveform synthesizer (NF Electronic Instruments, Model 1930, with a frequency accuracy of better than $5 \times 10^{-6}$ ) to the power amplifier that controls a large, electromagnetic exciter (Ling Dynamical Systems 200). Since the exciter is large with respect to the beam, the effect of the beam motion on the motion of the exciter is small.

The dynamics of the impacting beam is explored through bifurcation diagrams where the driving amplitude is used as the bifurcation parameter. Since the dynamical state depends very sensitively on the excitation amplitude, it is of crucial importance to measure and control the amplitude of the exciter motion very accurately. The driving amplitude is measured by making use of a Michelson interferometer, which comprises a small He-Ne laser (wavelength $\lambda=632.8 \mathrm{~nm}$ ) and a photodiode. The amplitude of the exciter motion is inferred from the number of interference fringes counted per period of the driving. This assumes that the motion is purely sinusoidal with a frequency equal to the driving frequency. The accuracy of the amplitude measurement is determined by the pickup of environmental mechanical vibrations. This background noise amounts to a fringe-passing frequency of approximately $80 \mathrm{~Hz}$, corresponding to an uncertainty in the amplitude measurement smaller than $0.3 \mu \mathrm{m}$. For the excitation amplitudes that are typically used, this amounts to an uncertainty better than 1 in 2500 .

The control of the driving amplitude is achieved with the help of a 12 bit digital voltage divider (Analog Devices AD
7548), which is driven by a computer. When the measured amplitude differs from the required value, the amplitude of the sine wave on the amplifier input is changed appropriately with a small fixed step (relative size smaller than $5 \times 10^{-5}$ ), which is smaller than the background noise. It needs to be so small because amplitude changes induce transient behavior of the oscillator that may irreversibly change the dynamical state of the impacting beam. This is especially important since we are interested in hysteretic transitions.

The deflection of the leaf spring is measured using a laser beam that is reflected off the upper part of the beam onto a position-sensitive detector (SL 76-1, UDT Sensors Inc.). For small beam deflections, the output voltage is linear in the beam displacement. The beam deflection is measured synchronously with the excitation at a fixed phase delay. Thus, subsequent measurements are points in a stroboscopic phase plane.

It turned out that the frequency of free oscillations $f_{b}$ $=T^{-1}$ of the beam varied with the ambient temperature at a rate of $0.05 \mathrm{~Hz} /{ }^{\circ} \mathrm{C}$. Therefore, the temperature of the entire setup was controlled to within $0.05^{\circ} \mathrm{C}$, so that $f_{b}$ remained constant to within $2.5 \times 10^{-3} \mathrm{~Hz}$. The temperature sensitivity is caused by the temperature dependent properties of the damping material and by the thermal expansion of the beam. Because the beam is excited close to resonance, the amplitude is strongly dependent on the driving frequency. Therefore, it is important to keep $f_{b}$ constant in precise experiments. The resulting overall stability of the experiment is such that the excitation amplitude at the first grazing bifurcation, which is the reference point for the bifurcation diagrams, could be reproduced to within the pickup noise ( 0.3 $\mu \mathrm{m})$ of the interferometer

The experimental oscillator differs from a simple harmonic oscillator with two degrees of freedom in the sense that it is a continuous system, so that many modes may be excited (transverse modes as well as torsional ones), where each mode has its own characteristic resonance frequency and damping coefficient. In addition, the relation between the deflection of the beam and the restoring force may be nonlinear due to its peculiar, albeit useful, geometry. We will nevertheless describe the oscillator with just three parameters, namely, the eigenfrequency of the undriven beam, its damping, and the restitution coefficient. An extension with a fourth parameter describing the resilience of the contact upon collision will be considered briefly.

Assuming that the beam response is represented by Eq. (1), the period $T$ and the damping coefficient $\lambda$ can be measured in a straightforward manner from the decaying oscillations of the unexcited beam,

$$
x(t) \sim e^{-\lambda t} \cos (2 \pi t / T+\phi) .
$$

The parameters $\nu$ and $\Omega^{2}$ in Eqs. (3) and (4) then follow trivially as

$$
\nu=\frac{2 \lambda}{f} \quad \text { and } \Omega^{2}=\left(\frac{2 \pi}{\xi}\right)^{2}+\frac{\nu^{2}}{4},
$$

with the parameters of the impact mapping 


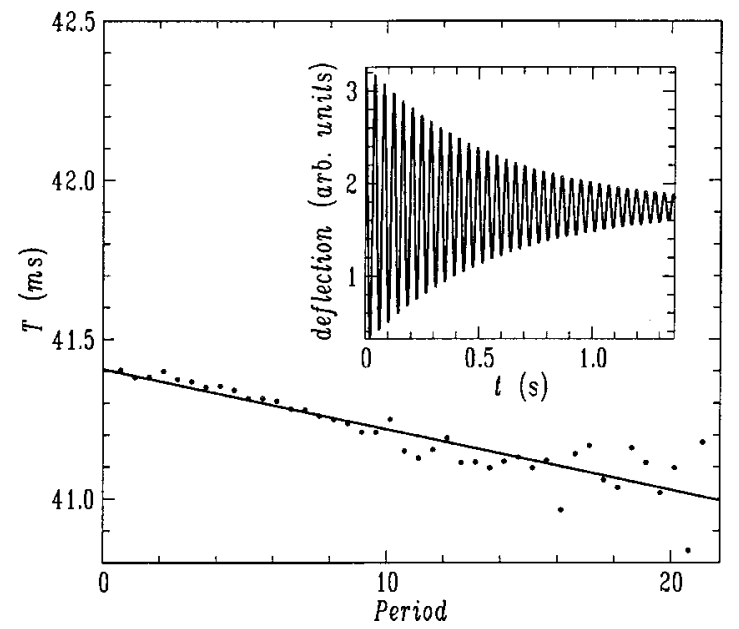

FIG. 4. Dots: duration of successive periods in a measured decaying oscillation. Each period length is measured from three successive zero crossings of the signal. The full line is a linear fit. It intersects the time axis at $41.41 \mathrm{~ms}$, which we take as the period of the beam. Inset: decaying oscillation from which both the period and damping constant (in Fig. 5 below) were measured.

$$
\gamma=e^{-\nu} \quad \text { and } \alpha=2 \gamma^{1 / 2} \cos \left(\frac{2 \pi}{\xi}\right)
$$

in terms of the reduced driving frequency $\xi=f T$ with $f$ the physical driving frequency. The damped oscillations are measured by suddenly switching off the exciter when the beam is in a stationary, nonimpacting orbit that nearly hits the constraint. The instantaneous position of the beam is measured with a sample frequency of $6 \mathrm{kHz}$ during $2.33 \mathrm{~s}$.

After subtraction of the equilibrium position, the period of the oscillations is found from the zero crossings of the signal, which are determined precisely by (linear) interpolation. Figure 4 shows a typical result for the period lengths. The time increments are observed to decrease with the amplitude of the decaying oscillation, indicating a weak nonlinearity of the beam. Because grazing impact dynamics corresponds to large beam deflections, we take the effective period $T$ of free oscillations as the extrapolation of the time increments in Fig. 4 to time 0 . This gives $T=41.41 \mathrm{~ms}$.

The coefficient of damping $\lambda$ is found from the maxima and minima of the measured relaxation curve. The value of each extreme is computed by fitting a parabola to the 17 data points that lie symmetrically around it. In Fig. 5 these values are plotted versus time. The damping coefficient is found from the exponential decay, $\lambda=1.780 \pm 0.005 \mathrm{~s}^{-1}$.

We will describe the collisions in our experiment by the coefficient of restitution model, which assumes that at impact the oscillator rebounds with a reduced velocity. The energy loss at impact is expressed by the coefficient of restitution: if $v_{c}$ is the collision velocity, the rebound velocity is $-r v_{c}$, with $0 \leqslant r \leqslant 1$. This is an admittedly crude description. In our case the energy dissipation at impact is caused by the excitation of many high-order modes which are quickly damped, for example, by the radiation of sound. We therefore interpret $r$ as an effective coefficient of restitution, which may differ from the one associated with collisions between a ceramic ball and hardened steel.

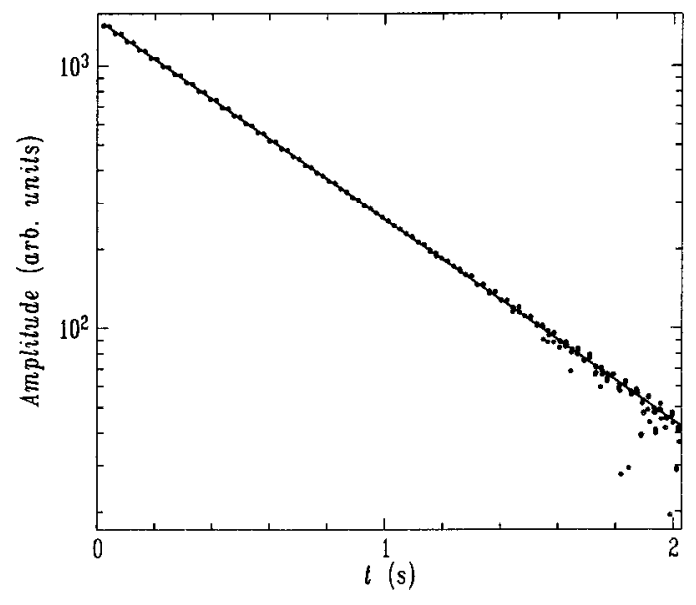

FIG. 5. Dots: decay of the extrema of a measured damped oscillation shown in the inset of Fig. 4. Dashed line: fit of exponential decay with decay constant $\lambda=1.78 \mathrm{~s}^{-1}$.

Figure 6 shows a typical time trace of the deflection of an undriven beam that was released from such a height that after about half an oscillation a single impact occurred. It is obvious that a multitude of higher-order modes is excited upon impact. An estimate of the effective coefficient of restitution was reached as follows. At long times, when the higher-order modes have damped out, it is possible to represent the data with Eq. (16) and determine the phase in a least squares procedure. The resulting expression for $x(t)$ can then be extrapolated back to the instant of impact. From Fig. 6 it is clear that the impact occurs very close to the turning point of the extrapolated solution. We therefore conclude that the coefficient of restitution is close to 0 and we take $r=0$. Further support for this effective value will be presented below. The excitation of higher-order modes is caused by the deformation of the beam at impact. In the simple model of Fig. 1, this is equivalent to a collision with a yielding wall. A similar two-spring model for hard collisions was studied both experimentally and theoretically by Shaw [10].

We study the dynamics of the impacting beam by means of bifurcation diagrams in which the forcing amplitude is used as control parameter and the forcing frequency is kept

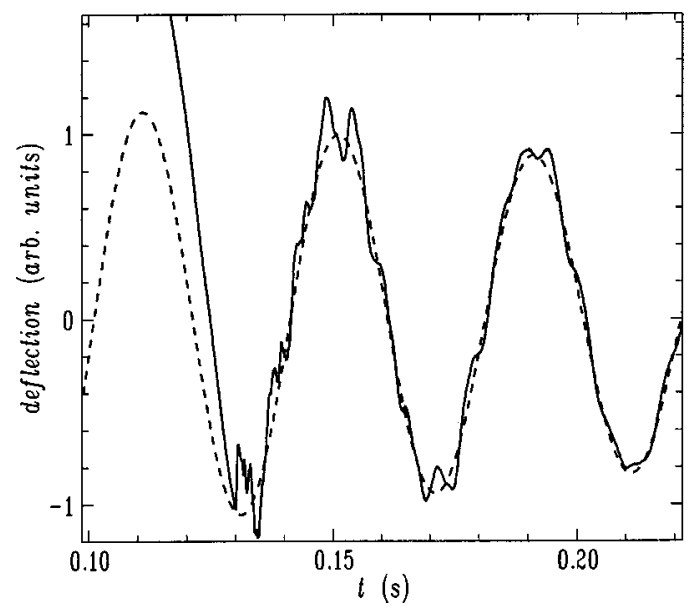

FIG. 6. Full line: time trace of the undriven beam which impacts at $t=0.13 \mathrm{~s}$. Dashed line: fit of damped oscillations in the time interval $0.25<t<1.3 \mathrm{~s}$ where the secondary oscillations have damped out. The fit is extrapolated back to the instant of impact. 

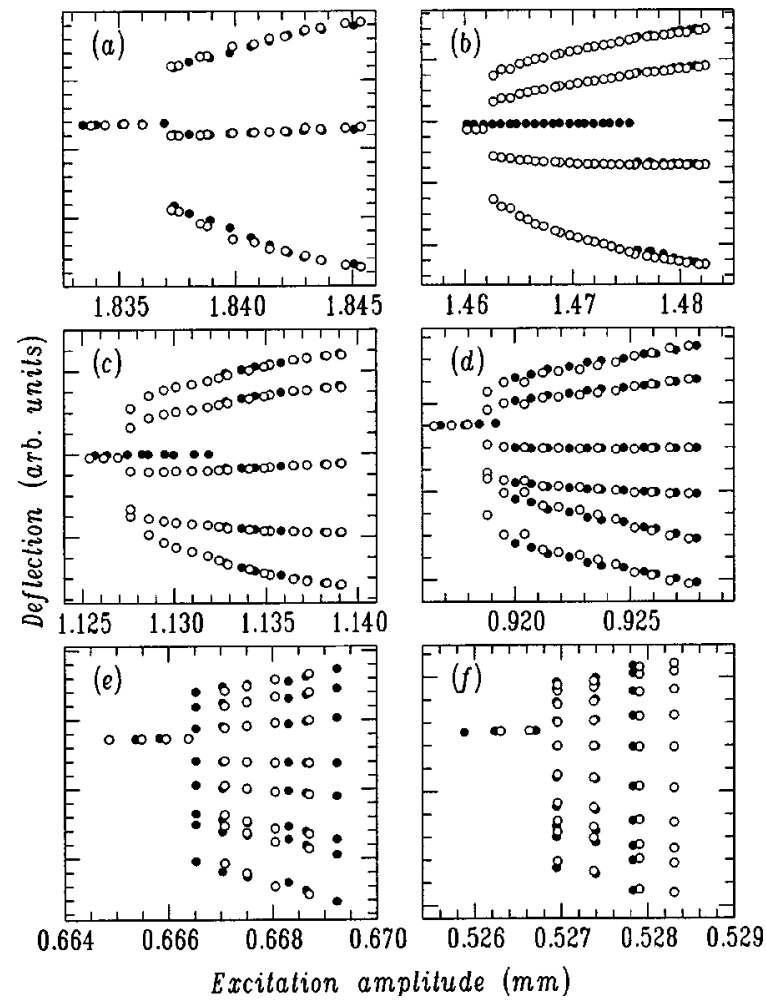

FIG. 7. Experimental bifurcation diagrams of $p_{1} \leftrightarrow p_{M}$ transitions with $M=3,4,5,6,8,10$ for (a) through (f), respectively. The closed dots are for the upward scan of the excitation amplitude; the open circles are for the downward scan. For this experiment the period of the free swinging beam is $T=40.80 \pm 0.02 \mathrm{~ms}$ and damping $\lambda=2.1 \pm 0.05 \mathrm{~s}^{-1}$. The excitation frequencies are $f=20.90$, $21.50,22.20,22.60,23.05$, and $23.40 \mathrm{~Hz}$ for (a) through (f), respectively.

constant. A measurement of the bifurcation diagram is started at a small driving amplitude with the beam in a stationary, nonimpacting orbit. The driving amplitude is then increased with small steps until the desired value is reached. As we are interested in hysteretic transitions, we make sure that the amplitude changes strictly monotonically. The step size in the amplitude can be as small as $0.8 \mu \mathrm{m}$, but it is larger when performing scans over large amplitude intervals. The oscillator is allowed to settle for approximately 100 forcing cycles during which the amplitude is maintained at its setpoint. Next, the beam deflection is registered for approximately 80 driving cycles. The phase of this stroboscopic measurement relative to the sinusoidal forcing signal is chosen such that the branches of the subharmonic orbits that appear in the diagrams do not overlap. The amplitude of forcing is increased up to approximately $10 \%$ above the excitation amplitude of the first grazing bifurcation. It is subsequently decreased until the impacting motion disappears and the oscillator settles in a nonimpacting orbit, which may be at an excitation amplitude well below the excitation amplitude of the first grazing collision.

\section{EXPERIMENTAL RESULTS}

An overview of measured period-adding transitions $p_{1} \leftrightarrow p_{M}$ from the nonimpacting state to maximal periodic orbits with period $M=3$ to 10 is shown in Fig. 7. For in-

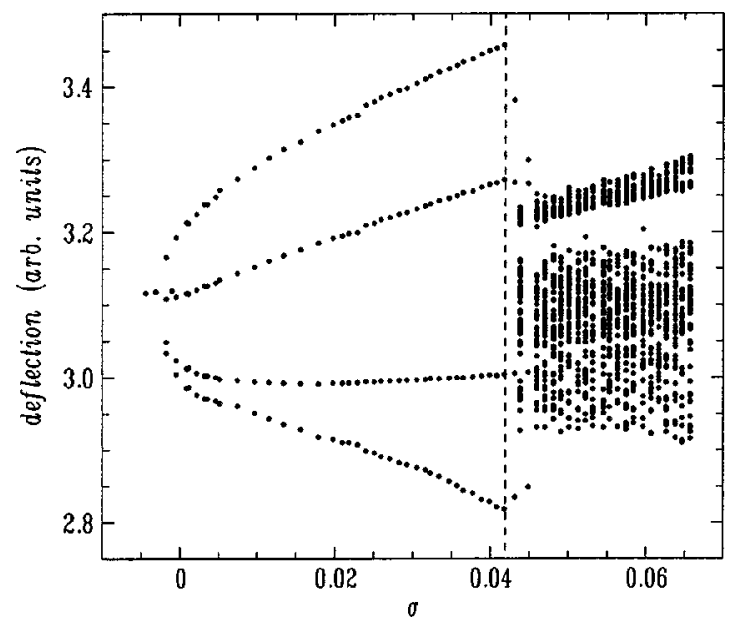

FIG. 8. The grazing bifurcation of a maximal $p_{M}$ orbit into a $p_{M / 2}$ orbit (two impacts per period) at the vertical dashed line is clearly observed in the bifurcation diagram as a sharp change in one of the measured positions at $\sigma \approx 0.042$ in the upward scan of $\sigma$. The excitation frequency is $f=21.35 \mathrm{~Hz}$.

creasing period lengths, the scale of the driving amplitude scan rapidly diminishes. The highest observed period $\left(p_{M=10}\right)$ is at the limit of our experimental resolution and stability. For this figure the (reduced) excitation frequency $\xi$ is set somewhere in the interval $\xi_{2}<\xi<\xi_{3}$ [Figs. 2(a) and 2(b)] so that the transition at $\rho=0$ is always $p_{1} \rightarrow p_{M}$. As can also be observed, the apparent hysteresis may vary wildly. In all cases the amplitude scans stop before the period- $M$ maximal orbit loses stability through an additional impact and becomes a period- $M$ orbit with two impacts $\left(p_{M / 2}\right)$.

The transition $p_{M} \rightarrow p_{M / 2}$ is found from the time traces of the recorded spring deflection. It gives rise to the characteristic jump in the bifurcation diagram which is illustrated for

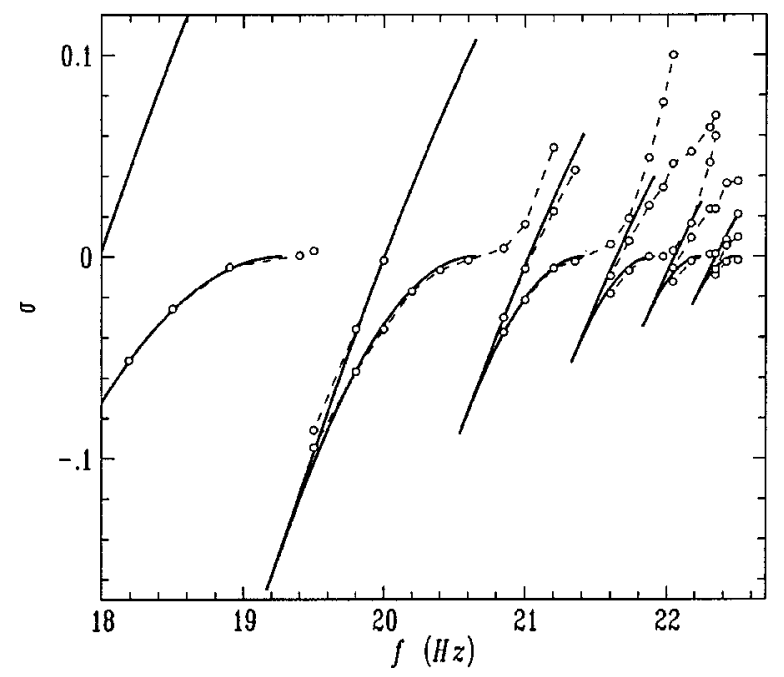

FIG. 9. Open circles: measured boundaries of stability tongues of maximal period- $M$ orbits, where $M=2-7$. At the lower boundary the transition $p_{M} \rightarrow p_{1}$ is observed in a downward scan of $\sigma$. At the upper boundary a second impact $p_{M} \rightarrow p_{M / 2}$ occurs. The measured upper and lower boundary points for each $P_{M}$ tongue are connected by dashed lines. Full lines: tongues as computed from the differential equation for $T_{1}=41.5 \mathrm{~ms}, \lambda=1.78 \mathrm{~s}^{-1}$, and $r=0$. 


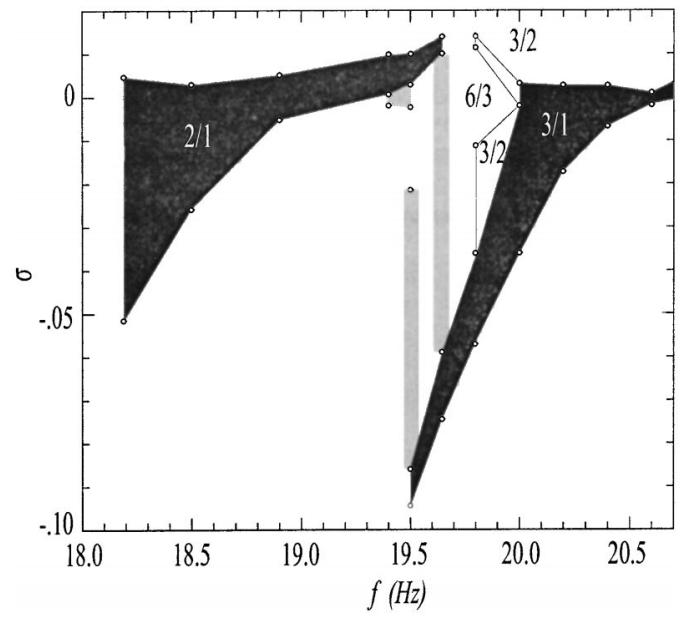

FIG. 10. Measured bifurcation diagrams near maximal periodic orbits with $M=2,3$. The diagram is composed from amplitude scans at discrete frequencies. The dots at the largest $\sigma$ are the maximal amplitude in each scan. The black regions are stability tongues of maximal $p_{M}$ orbits; the gray regions are chaotic states.

the $p_{4} \rightarrow p_{4 / 2}$ transition in Fig. 8. The additional impact occurs at the turning point that immediately precedes the primary impact, as this turning point is closest to the wall. At the bifurcation, the motion between these two impacts changes most, and the change in the corresponding stroboscopic branch of the bifurcation diagram is largest. The other branches are affected less as they are registered after the relatively hard primary impact.

In Fig. 9 the measured hysteresis and secondary bifurcation of the maximal periodic orbits are compared with the stability tongues computed from the differential equation Eqs. (3) and (4). In the computation we have used the parameter values $T=41.5 \mathrm{~ms}, \lambda=1.78 \mathrm{~s}^{-1}$, and $r=0$. The period of free vibrations $T=41.5 \mathrm{~ms}$ is chosen slightly larger than the period $(T=41.41 \mathrm{~ms})$ that was measured from the decaying oscillations. In this way the frequency windows in which the maximal orbits are observed fit well with the predicted ranges. The frequency where the computed maximal orbits $M=2,3 \ldots$ are found depends strongly on the used $T$ of the model. The damping constant $\lambda=1.78 \mathrm{~s}^{-1}$ is taken from the relaxation measurements. The width of the stability tongues depends very sensitively on the restitution coefficient $r$. The tongues shrink to zero width as $r \rightarrow 1$. The best fit between the theory and experiment was obtained for $r$ $=0$, which agrees with the effective value of $r$ that was estimated from time traces of impacting orbits in Sec. II. The conclusion is that the observed bifurcation sequence can be reproduced well using only three parameters to describe the experiment. We emphasize that we view these parameters as effective values, reflecting the influence of higher-order modes that are excited at impact.

The experimentally observed dynamics in the vicinity of the first grazing bifurcation and beyond is summarized in Figs. 10 and 11. The windows in which maximal periodic orbits $p_{M}$ are found cover a large part of parameter space and have a similar shape for $M=4-7$ (the $M=2$ and 3 windows are only partly measured), whereas their size decreases when $M$ increases. The upper boundary due to a second impact meets the lower boundary at the low-frequency side of the maximal $M$-periodic stability tongue. The very tip is not al-

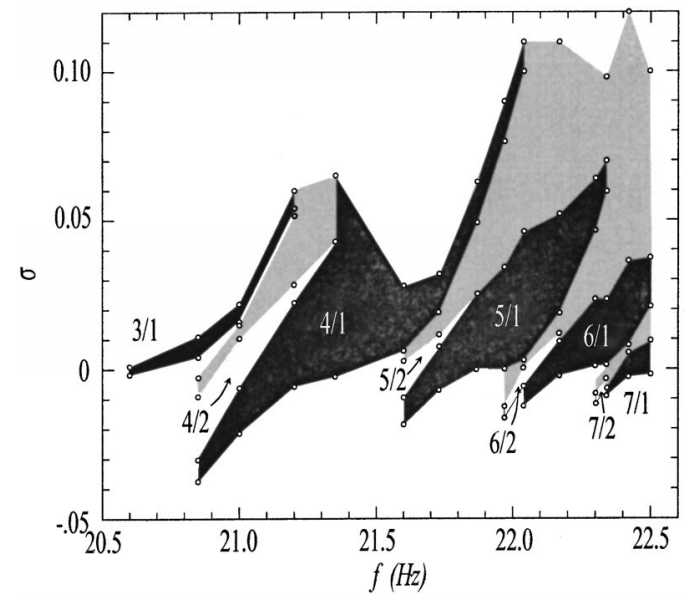

FIG. 11. Measured bifurcation diagrams near maximal periodic orbits with $M=3-7$. The diagram is composed from amplitude scans at discrete frequencies. The dots at the largest $\sigma$ are the maximal amplitude in each scan. The black regions are stability tongues of maximal $p_{M}$ orbits; the gray regions are chaotic states.

ways resolved in the experiment. The tip can extend as much as $10 \%$ below the driving amplitude where impacts first occur in a smooth upward scan of $\sigma$. Figures 10 and 11 also illustrate that the boundaries of the regions of maximal periodic orbits are slightly more complicated than was stated so far. The simultaneous creation of a pair of stable and unstable $M$-cycles (and the associated hysteresis) occurs for $\sigma$ $\leqslant 0\left(\xi \leqslant \xi_{3}\right.$ in Fig. 2). For scaled frequencies $\xi>\xi_{3}$ there is often a transition to chaos at $\sigma=0$ which yields to the $M$-cycle at some positive value of $\sigma$. Of course, this part of the boundary was missed in the computed stability diagrams.

The occurrence of an additional collision for increasing $\sigma$, which signifies the upper boundary of the stability tongue, can either lead to a $p_{M / 2}$ orbit (at the low-frequency side of the tongue) or turn directly into chaos at the high-frequency side. When the driving amplitude is further increased, the $p_{M / 2}$ orbit changes into chaos. The regions in the $\xi, \sigma$ parameter plane where the $p_{M / 2}$ orbit is found form a geometrically converging sequence for $M=4-7$, analogous to the stability tongues of the maximal $p_{M}$ orbits.

Chaotic motion is observed between maximal orbits and can also be hysteretic. For example, at $f=21.35 \mathrm{~Hz}$ an $M=4$ maximal periodic orbit bifurcates to chaos via a $p_{M / 2}$ orbit in the upward scan of $\sigma$, whereas in the downward scan the chaotic motion bifurcates directly into the $p_{M}$ orbit. Apparently, a chaotic attractor and a periodic orbit coexist in some regions of parameter space, and it depends on how this region is approached, and possibly on the level of mechanical disturbances, on which attractor the oscillator will settle.

In order to further illustrate the richness of the bifurcation structure near grazing, we present in Figs. 12 and 13 measured bifurcation plots at a driving frequency $f=21.60$ and $21.97 \mathrm{~Hz}$, respectively. We contrast the measured bifurcations with the results of numerical simulations. The frequencies correspond to the transitions $p_{M=5} \rightarrow p_{1}$ and $p_{M=6}$ $\rightarrow p_{1}$, respectively. However, in the latter case these maximal periodic orbits cannot be reached through smooth scans of the driving amplitude and it is necessary to disturb the oscillator. In Fig. 12 the first impacting state encountered at $\sigma=0$ is the $M=\frac{5}{2}$ maximal orbit, with two impacts per period. This state turns into chaos at larger $\sigma$, which yields to 

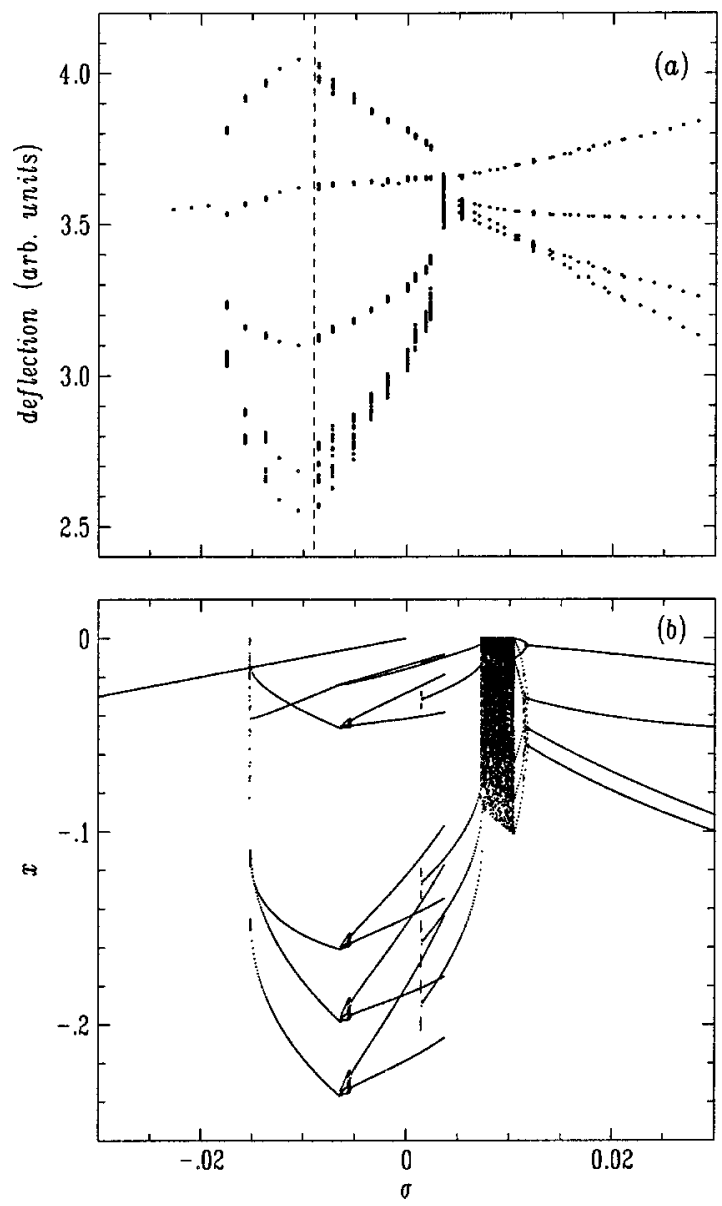

FIG. 12. Bifurcation diagrams of the impact oscillator at a driving frequency $f=21.60 \mathrm{~Hz}$. (a) Measured diagram. The dashed line indicates the $p_{4} \rightarrow p_{4 / 2}$ transition. (b) Computed bifurcation diagram. The first impacting state encountered at $\sigma=0$ is the $M$ $=5 / 2$ maximal orbit, with two impacts per period. This state turns into chaos at larger $\sigma$, which yields to the $M=4$ periodic orbit. The $M=5$ state and the $p_{5} \rightarrow p_{1}$ transition are reached only on the downward scan, once the upper boundary due to second impacts has been passed at $\sigma=-0.18$.

the $M=4$ periodic orbit. The $M=5$ state and the $p_{5} \rightarrow p_{1}$ transition are reached only on the downward scan, once the upper boundary due to second impacts has been passed at $\sigma=-0.18$. Both experiment and numerical simulation display the same course of events, but fine details in the simulation for $\sigma \in[-0.006,0.0035]$ remain unresolved in the experiment. Of course, the shape of the phase diagram is also determined by the stroboscopic phase, which cannot be compared for the two cases.

Starting from the nonimpacting state in Fig. 13, a smooth upward scan of the driving amplitude leads to the small chaotic attractor at $\sigma=0$, which in turn is followed by an $M=5$ maximal periodic orbit and chaos. In the subsequent downward scan, the nonimpacting state is reached again at $\sigma=0$. The maximal $M=6$ periodic orbit with the chaotic attractor to which it bifurcates through a second impact can be reached only by hitting the oscillator. Exactly the same phenomenon is found in the numerical bifurcation diagram, where the hit is done by setting $x$ to a large value. The overall structure of the computed diagram is very similar to
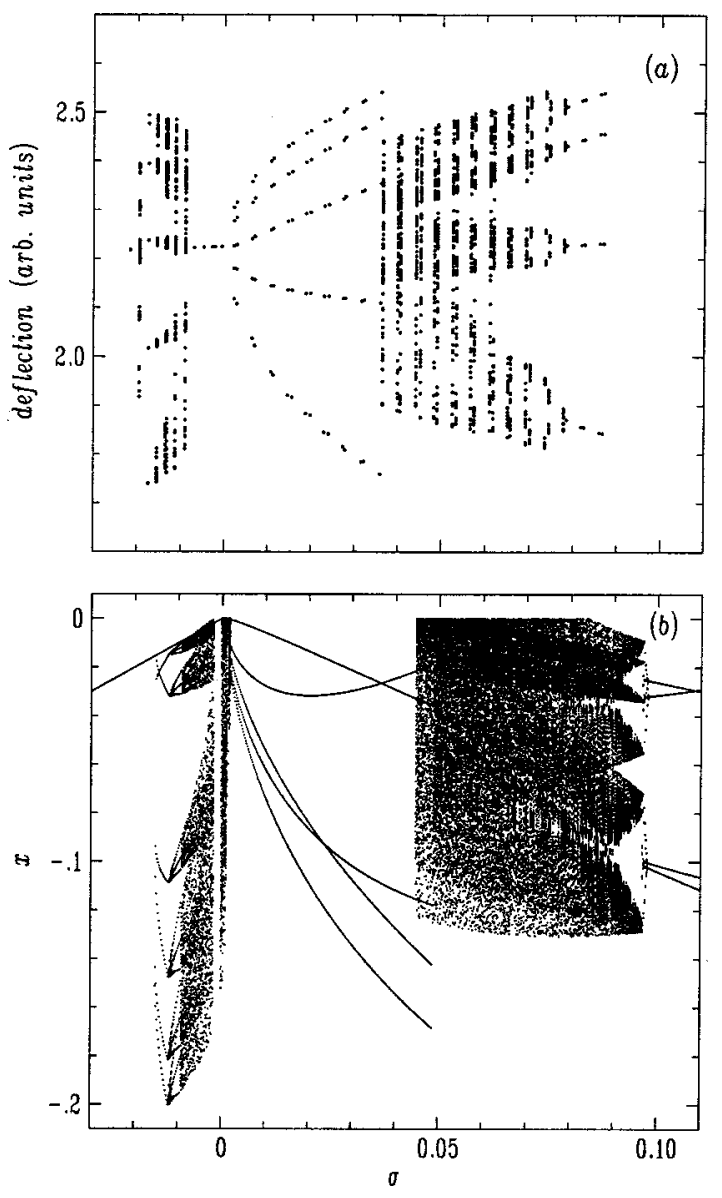

FIG. 13. Bifurcation diagrams of the impact oscillator at a driving frequency $f=21.97 \mathrm{~Hz}$. (a) Experiment. In the upward scan of the driving amplitude the states $p_{5 / 1}$, chaos, and $p_{4 / 1}$ are encountered; in the downward scan the sequence is $p_{4 / 1}$, chaos, $p_{5 / 1}$, chaos, $p_{6 / 2}$. (b) Computed bifurcation diagram with a similar sequence of states as observed in the experiment. In the experiment the impacting states at negative $\sigma$ could be reached only by hitting the oscillator; in the numerical simulation this was done by suddenly setting $x$ to a large value.

the experimental one. Both experiment and simulation have the disconnected $p_{M=6}$ and chaotic states at $\sigma<0$; they share the $p_{M=5}$ orbit followed by a large region of chaos and the $p_{M=4}$ cycle. Details differ, however, especially the structure of the large chaotic state at $\sigma>0.043$. We conclude that the richness of the observed bifurcations near grazing is quite well captured by the numerical simulation, which models the experiment with only three (effective) parameters.

We emphasize that our experiment is far from ideal as many high-order modes are excited upon impact. Apparently, transitions near grazing orbits are forgiving about this nonideality. A possible explanation is the presence of long time delays between subsequent impacts, which get longer as $M$ increases. This lets higher modes damp out. For states that have a more rapid succession of impacts, for example, $p_{M / 2}$ periodic states, we found larger discrepancies between measured and computed bifurcation diagrams. In these cases the time for higher modes to decay is shorter. We conclude that grazing bifurcations are not sensitive to experimental detail and are therefore a universal phenomenon. 


\section{TESTING GRAZING IMPACT MAPS}

The power of grazing impact maps is the reduction of grazing dynamics to the application of a map. A key feature of these mappings is the presence of a square-root singularity. It was shown by us that such a singularity survives soft impacts with a yielding wall [7].

The details of these mappings, in particular the relation of their coefficients to the parameters of the physical system and the relation between the bifurcation parameter $\rho$ and the reduced driving amplitude $\sigma$, depend on the details of the analysis of grazing orbits in the differential equation. This analysis is not a trivial task. Therefore, it is interesting to make a quantitative comparison between the prediction of mappings and the solutions of the differential equation. We will make this comparison for the stability tongues of the sequence of maximal periodic orbits that was also studied experimentally. In our comparison we have not exhaustively scanned parameter space, but instead have used the physical parameters of the experiment $\left(T=41.5 \mathrm{~ms}, \lambda=1.78 \mathrm{~s}^{-1}, r\right.$ $=0)$. The predictions of the soft map will be tested against the differential equation using $\Gamma=10$ and $r=0.5$.

Finding the stability tongues in the maps was done in a simple numerical scheme using Eq. (15). Once the stable impacting root at the lower boundary $\rho=-\rho_{M}$ of the resonance tongue is located, it is followed for increasing $\rho$ until a second collision at iterate $M-1$ occurs. The upper boundary can then be determined accurately using a zero finding procedure. We have used a similar procedure for finding the stability boundaries of the resonance tongues of the differential equation Eq. (3). When collisions are with a stiff wall, a simple analytical expression exists for the lower boundary [13]. The upper boundary was found analogously to that of the map. For tracing the stability boundaries of maximal $M$-periodic orbits in the differential equation for the soft wall, we have not sought analytical solutions but determined the boundaries in an experimental fashion by using amplitude scans at many frequencies. In all cases the stability boundaries are expressed in terms of the reduced driving strength $\sigma$, which is the relevant physical variable.

A comparison between the maximal $M$-periodic stability tongues computed from the Nordmark map Eqs. (5),(6) and those computed directly from the differential equation Eq. (3) is shown for hard collisions in Fig. 14(a). The Nordmark map predicts the lower boundary of the stability tongues quite well, but it grossly overestimates the driving amplitude at which the additional impact occurs. The comparison with the map derived by us [Eqs. (10),(11)] is shown in Fig. 14(b). At the used value $r=0$, a more favorable agreement with the differential equation is found.

We have argued that for increasing period $M$ the conditions for deriving maps become increasingly favorable, and the agreement between the map and the differential equation result should improve. This is observed for the map Eqs. (10),(11), but not for the Nordmark map Eqs. (5),(6). Most probably this difference is caused by the term proportional to $x_{n}$ in Eq. (10), wich is absent in the Nordmark map. For increasing restitution coefficient $r$ the width of the tongues in the differential equation becomes small, but the map Eqs. (10),(11) predicts that tongues with $M<\tilde{M}$ disappear altogether $(\tilde{M} \approx 4$ for $r=0.5)$. This is in obvious disagreement
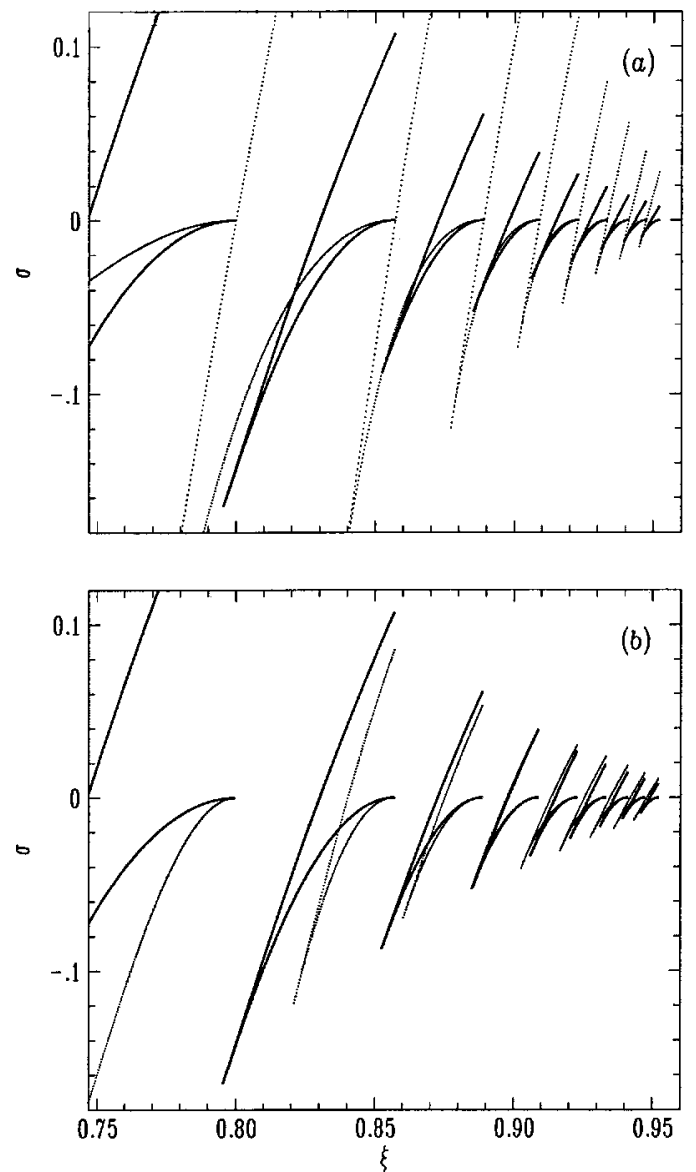

FIG. 14. Comparison of stability tongues of maximal periodic orbits in the mapping and the differential equation. The parameters used are $T=41.50 \mathrm{~ms}, \lambda=1.78 \mathrm{~s}^{-1}$, and $r=0$. Shown are the tongues with $M=2$ (leftmost) to $M=10$ (rightmost). (a) Dotted line: stability boundaries computed from the Nordmark map Eqs. (5),(6). Full line: as computed from the differential equation Eq. (3). (b) Same as (a), but now the dotted line is the prediction of a map that was derived by us [Eqs. (10),(11)]. The upper boundary of the $M=2$ tongue is missed in this map.

with the tongues computed from the differential equation Eq. (3). Clearly, in some regions of parameter space the mappings are closer to the original dynamics than in other regions.

Maximal periodic resonance tongues for collisions with a yielding wall are shown in Fig. 15. In this case the Nordmark map does not apply, and we compare the prediction of the map Eqs. (10),(11) with the result of the numerical simulation of Eq. (3). The parameters used are $T=41.50 \mathrm{~ms}, \lambda$ $=1.78 \mathrm{~s}^{-1}$, and $r=0.5$, and $\Gamma=10$. We have not used sophisticated path following techniques, but the boundaries of the $M$-periodic resonance tongues in the differential equation were found from simple scans of the driving amplitude, much as in the experiment.

The question of whether a map is soft or hard depends on the parameter $\beta=r v_{c} \Gamma^{1 / 2} \Omega / 4 \pi^{2}$. Soft collisions are the case if $\beta \ll 1$, and the map Eqs. (10),(11) with the substitution $r$ $\rightarrow-r$ applies. Hard collisions are the case if $\beta \gg 1$ and the map remains unchanged. The map Eqs. (10),(11) does not apply in intermediate cases, so collisions must be either hard or soft. The problem is that in a scan of the bifurcation diagram from small to large $M$, the impact velocity $v_{c}$ decreases and collisions may turn from hard to soft. Let us suppose that 


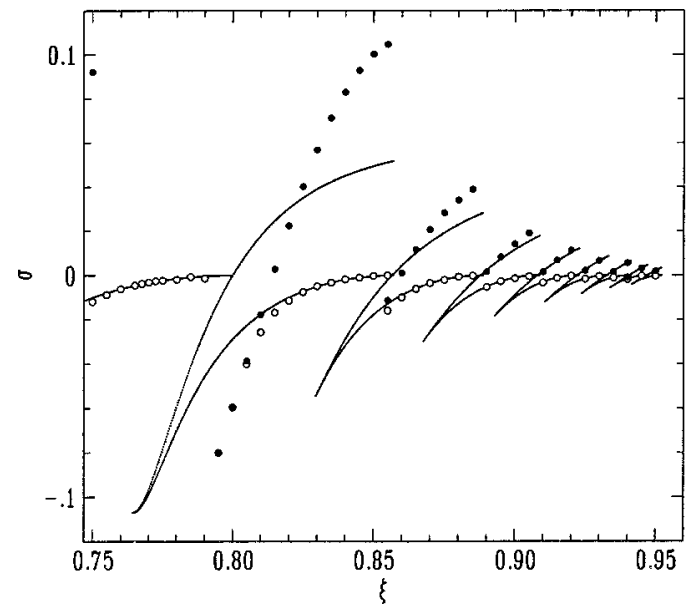

FIG. 15. Comparison of stability tongues of maximal periodic orbits in the mapping and the differential equation for collisions with a yielding wall. The parameters used are $T=41.50 \mathrm{~ms}, \lambda$ $=1.78 \mathrm{~s}^{-1}$, and $r=0.5$. Shown are the tongues with $M=2$ (leftmost) to $M=10$ (rightmost). Dotted line: prediction of the map Eqs. (10),(11) for soft collisions with a soft wall (with the change $r$ $\rightarrow-r)$. The symbols are computed from the differential equation Eq. (3) with $\Gamma=10$. Open circles: lower boundary of resonance tongues. Closed dots: upper boundary.

during a collision only the force between the wall and the mass changes and that the other forces remain constant. Then, for soft impacts the time of residence $\Delta t$ in the wall is a linear function of the collision velocity and it is a constant for hard collisions.

Figure 16 shows the dependence of the time delay $\Delta t$ on the collision velocity $v_{c}$ for two scaled frequencies, one $(\xi$ $=0.805)$ near a low-order resonance $(M=3)$ in Fig. 15 , the other one $(\xi=0.95)$ near $M=10$. The time delays were registered while scanning a bifurcation diagram. Clearly, the $M=3$ tongue is not yet soft, so the soft map does not strictly apply here and the discrepancy between the stability boundaries of the map and the differential equation is largest.

The tongues that were computed from the soft impact

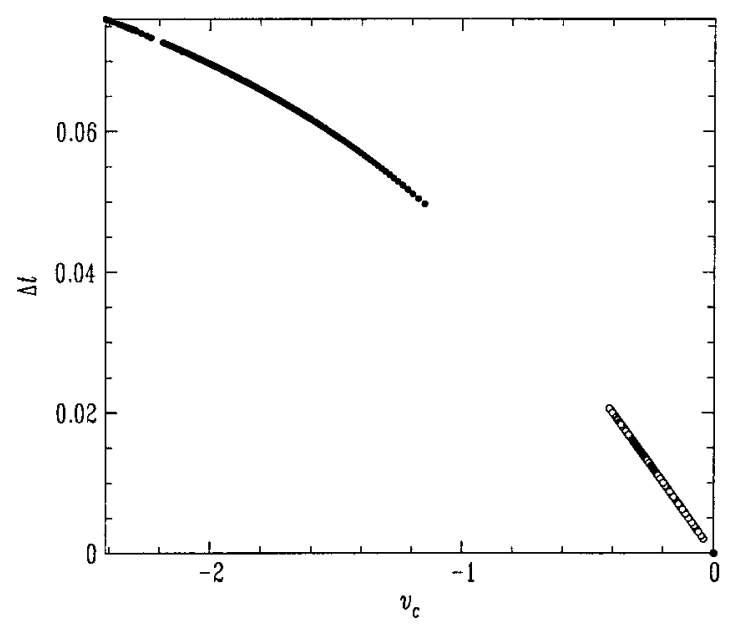

FIG. 16. Time delay experienced in a collision with a soft wall as a function of the collision velocity. Closed dots: scaled frequency $\xi=0.85$ near a $M=3$ maximal periodic orbit. Open circles: $\xi$ $=0.95$ near $M=10$. Soft collisions are the case if $\Delta t$ depends linearly on $v_{c}$; this is apparently not yet the case near the $M=3$ orbit.

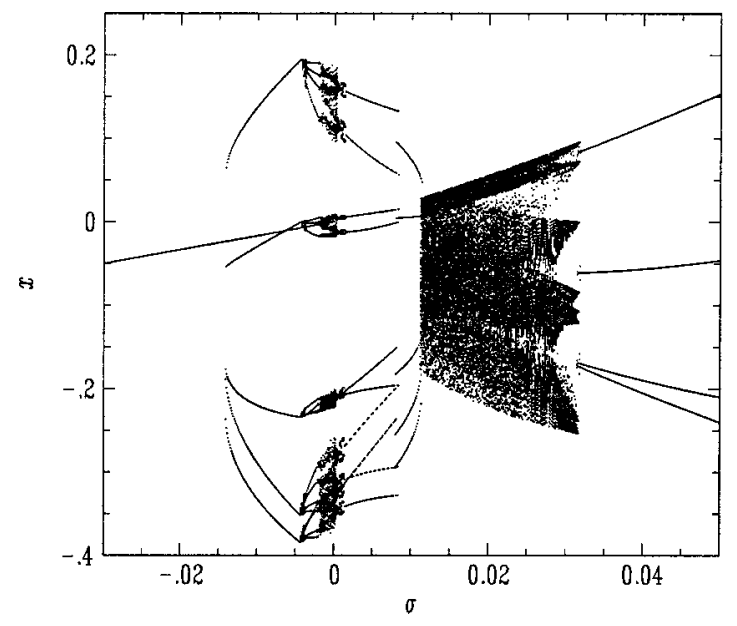

FIG. 17. Bifurcation diagram of the map Eqs. (10),(11) for hard collisions at a driving frequency $f=21.60 \mathrm{~Hz}(\xi=0.8964)$. At $\sigma$ $\approx-0.014$ the transition $p_{5} \rightarrow p_{1}$ can be observed. The structure of the diagram can be compared to Fig. 12 as it displays the same sequence of states, but we point out a change of the horizontal scale.

map Eqs. (10),(11) display "overhang:' at some driving frequencies both $M$ and $M-1$ periods are stable, with the $M$ -1 tongue hanging over the $M$ tongue. In these cases the $M$-periodic cycles are inaccessible in experiments where the excitation amplitude is smoothly varied. Therefore, the overhangs are not completely resolved in the numerical simulation of the differential equation (where stability boundaries were found "experimentally"). The agreement between the tongues computed from the soft map and those computed from the differential equation is quite reasonable.

At large driving amplitudes where we find orbits far beyond grazing ones, the validity of the maps is obviously challenged. Still, the general bifurcation structure is reproduced remarkably well by maps. Figure 17 shows iterates of the map Eqs. (10),(11) for collisions with a hard wall at a driving frequency $f=21.60 \mathrm{~Hz}$. The map displays a similar sequence of states as the differential equation in Fig. 12. For increasing $\sigma$ we find in both cases a period-10 orbit at $\sigma$ $=0$, followed by a period- 5 cycle, chaos, and a period- 4 orbit; with of course at negative $\sigma$ the $p_{5} \rightarrow p_{1}$ transition. For large driving strengths a shift in $\sigma$ is apparent. For the parameters used, the Nordmark map Eqs. (5),(6) predicts a scenario that qualitatively disagrees with both experiment and differential equation.

We have modeled the experiment with an effective restitution coefficient $r=0$. For such completely dissipative collisions, the wall resilience, of course, does not matter. The question is if the experiment can be modeled using a larger $r$, which is more realistic for the instantaneous collision, but allowing for a finite wall stiffness. We recall that the main reason to choose $r=0$ is to match the width of the computed $M$-tongues in Fig. 9 with those of the experiment. At larger $r$, the tongues become too narrow, but this can be cured by allowing for a finite wall stiffness. Our preliminary conclusion is that this procedure does not lead to satisfactory agreement with the experiment. Soft impacts result in a bifurcation diagram that is essentially different from hard collisions, for example, in comparison with Fig. 14, the resonance 
tongues of soft collisions in Fig. 15 have been shifted to lower frequencies.

\section{CONCLUSION}

Grazing impact bifurcations are the consequence of a square-root singularity. We have demonstrated that an experiment, despite its nonidealities, can be effectively modeled as a singular system. We have indicated why these nonidealities, such as the multimode character of impacts, do not spoil such a description. Because of this singularity, and the associated strong distortion of phase space, low-velocity impacts may lead to richer dynamics than hard collisions $[14,15]$.

In the context of maps we have shown explicitly that the square root also survives soft impacts with a soft wall. In this case it is essential to allow for a discontinuity in terms of a restitution coefficient $r<1$. For perfectly elastic soft collisions with a soft wall, however, the square root vanishes and collisions need to become hard again to restore it. It is therefore necessary to consider more realistic impact models. An interesting testing ground would be collisions on a mesoscopic scale in atomic force microscopy [16]. Such atomic impact oscillators will be studied in a forthcoming publication.

\section{ACKNOWLEDGMENTS}

We thank Doug Binks for many insightful discussions and for the program to compute the stability boundaries of the resonance tongues of the differential equation. We also thank Jan Niessen for technical assistance. We gratefully acknowledge financial support by the Nederlandse Organisatie voor Wetenschappelijk Onderzoek (NWO).
[1] F. Peterka and J. Vaciík, J. Sound Vib. 154, 95 (1992).

[2] A. B. Nordmark, J. Sound Vib. 145, 279 (1991); Phys. Rev. E 55, 266 (1997); M. H. Fredriksson and A. B. Nordmark, Proc. R. Soc. London, Ser. A 453, 1261 (1997).

[3] G. S. Whiston, J. Sound Vib. 118, 395 (1987); C. J. Budd and F. Dux, Nonlinearity 7, 1191 (1994); S. Foale and S. R. Bishop, Philos. Trans. R. Soc. London, Ser. A 338, 547 (1992); C. J. Budd, F. Dux, and A. Cliff, J. Sound Vib. 184, 475 (1995).

[4] W. Chin, E. Ott, H. E. Nusse, and C. Grebogi, Phys. Rev. E 50, 4427 (1994).

[5] Singularities in this system in a more general context are discussed by G. S. Whiston, J. Sound Vib. 152, 427 (1992).

[6] H. E. Nusse and J. A. Yorke, Physica D 57, 39 (1992); H. E. Nusse, E. Ott, and J. A. Yorke, Phys. Rev. E 49, 1073 (1994).

[7] J. Molenaar, J. G. de Weger, and Willem van de Water (un- published).

[8] J. de Weger, D. J. Binks, J. Molenaar, and W. van de Water, Phys. Rev. Lett. 76, 3951 (1996).

[9] M. G. Thompson, S. R. Bishop, and S. Foale, Mach. Vib. 3, 10 (1994).

[10] S. W. Shaw, J. Sound Vib. 99, 199 (1985).

[11] S. Foale, Proc. R. Soc. London, Ser. A 347, 353 (1994).

[12] M. Hénon, Physica D 5, 412 (1982).

[13] M. Senator, J. Acoust. Soc. Am. 47, 1390 (1970).

[14] H. Lamba, Physica D 82, 117 (1995).

[15] S. W. Shaw and P. Holmes, Phys. Rev. Lett. 51, 623 (1983); J. Sound Vib. 90, 129 (1983).

[16] Q. Zhong, D. Inniss, K. Kjoller, and V. B. Ellings, Surf. Sci. 290, L688 (1993); J. P. Spatz, S. Sheiko, M. Möller, R. G. Winkler, P. Reineker, and O. Marti, Nanotechnology 6, 40 (1995). 